\title{
Monocular Depth Ordering using Perceptual Occlusion Cues
}

\author{
Babak Rezaeirowshan, Coloma Ballester and Gloria Haro \\ Department of Information and Communication Technologies, Universitat Pompeu Fabra, Barcelona, Spain
}

Keywords: Monocular Depth, Ordinal Depth, Depth Layering, Occlusion Reasoning, Convexity, T-junctions, Boundary Ownership, 2.1D.

\begin{abstract}
In this paper we propose a method to estimate a global depth order between the objects of a scene using information from a single image coming from an uncalibrated camera. The method we present stems from early vision cues such as occlusion and convexity and uses them to infer both a local and a global depth order. Monocular occlusion cues, namely, T-junctions and convexities, contain information suggesting a local depth order between neighbouring objects. A combination of these cues is more suitable, because, while information conveyed by T-junctions is perceptually stronger, they are not as prevalent as convexity cues in natural images. We propose a novel convexity detector that also establishes a local depth order. The partial order is extracted in T-junctions by using a curvature-based multi-scale feature.Finally, a global depth order, i.e., a full order of all shapes that is as consistent as possible with the computed partial orders that can tolerate conflicting partial orders is computed. An integration scheme based on a Markov chain approximation of the rank aggregation problem is used for this purpose. The experiments conducted show that the proposed method compares favorably with the state of the art.
\end{abstract}

\section{INTRODUCTION}

Depth perception in humans enables a robust 3D vision even in the presence of a single view stimulus. Such a system is desirable in computer vision mainly due to its many applications and the abundance of monocular cameras. Human vision harnesses monocular cues to resolve inherent ambiguity caused by 3D to $2 \mathrm{D}$ projection in the image formation process and creates a sensible 3D perception. Monocular depth perception cues consist of dynamic cues and static cues. Dynamic cues, such as motion occlusion and motion parallax require multiple frames and motions in the scene as stimuli which are out of scope of this work. In this proposal, the focus is on static cues, namely, convexity and T-junctions; other cues in this category are perspective, relative dimensions, lighting and shadow.

While physiological aspects of these cues have been widely studied in the literature of psychophysics and vision, there is only a handful of research works that test these theories in a practical scenario using computer vision methods. Most of the work related to depth estimation in computer vision focuses on stereo disparity or motion parallax, both of which use triangulation to compute depth. While triangulationbased methods provide absolute depth, which is de- sirable in many applications, they require two or more views. Monocular static cues on the other hand, can be combined to create a depth perception in the absence of binocular and dynamic monocular cues or as a complement to improve existing depth perception in a much wider domain.

The goal of this paper is, given a single image from an uncalibrated camera and its decomposition in shapes (that are assumed to represent the projection of the 3D objects on the image plane; e.g., a segmentation), to create a globally consistent depth order of these shapes that constitute the image scene. For this purpose, occlusion cues between objects, namely T-junctions and convexities are used. Following the underlying assumption for extracting depth from occlusion cues, we assume that the image is composed of objects that are fronto-parallel to the camera. This is also referred to as the dead leaves model, a term coined by Matheron (Matheron, 1968), which constitutes a model for image formation where the image is made by objects falling on top of each others and partially occluding them. The reason for making such assumption is that in the presence of non fronto-parallel objects in the image, e.g. floor, occlusion does not translate to depth order (see Figure 1).

Given an image that satisfies the dead leaves model, the occlusion cues provide a depth order 

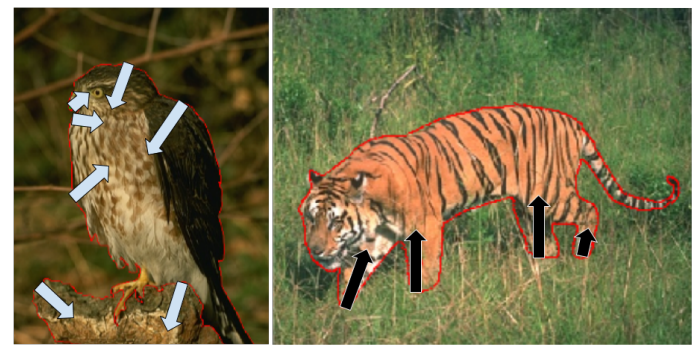

Figure 1: Dead leaves model (DLM) and correctness of convexity cues. The left image follows the DLM while the right one doesn't. Arrows indicate the occluding object suggested by convexity cues. Bright arrows indicate a correct depth order while dark arrows indicate a wrong one.

among neighbouring regions. However, we require a global order to establish a rough $3 \mathrm{D}$ model of the scene, which is understood here as obtaining a consistent global order from a number of partial orders, which may contain some discrepancies. This problem is in general referred to as rank aggregation and it has been dealt with in several fields of computer science (Dwork et al., 2001; Basha et al., 2012). This ordering problem appears whenever there are multiple operators providing partial orders with transitive relations. The goal is to use the transitivity to obtain a global robust order as consistent as possible with the partial orders. Transitivity between orders can be stated as the following property: if we have a partial order indicating $\mathrm{A}<\mathrm{B}$ and another one indicating $\mathrm{B}<\mathrm{C}$, thus we can infer the global order $\mathrm{A}<\mathrm{B}<\mathrm{C}$. Our approach stems from the fact that transitivity of local orders can be utilized to obtain a global order using rank aggregation.

Our main contributions in this paper are (i) a depth ordering system based on monocular perceptual cues that allows reasoning without need for camera calibration, multiple frames, or motion, (ii) a novel general convexity cue detector that assigns a local depth order based on convexity and which is based on the convex hull of a shape, and (iii) the extraction of a global depth order by a robust integration of the partial orders.

\section{RELATED WORK}

3D modeling has received a significant attention from the computer vision community, with studies focusing on various aspects of $3 \mathrm{D}$ perception. Due to the vastness of the literature in this field, we will focus on studies conducted on monocular static cues. Computational methods for depth extraction from a single image can be categorized into supervised methods and Gestalt-based methods. Supervised methods ei- ther learn depth directly from training images (Saxena et al., 2008; Eigen et al., 2014), or alternatively learn and detect cues (Chen et al., 2013; Jia et al., 2012), geometric (Hoiem et al., 2011) or semantic (Liu et al., 2010) information and use them to infer an absolute depth. Aforementioned approaches are aimed at a specific type of scene (either outdoor or indoor scenes) and require additional training or parameter tuning to achieve high performance on the unfamiliar scenes. Alternatively, other approaches have been suggested in the literature that use human perception and vision as the basis from which to attempt to infer a computational model simulating the known processes of human vision. Our work falls in the latter category. Thus, we focus on the use of T-junctions and convexity cues for establishing a depth order. The role of Tjunctions as a cue for recovering surface occlusion geometry was introduced by (Guzmán, 1968), and later stressed by (Malik, 1987; Rubin, 2001). Moreover, through the Gestalt school of thought in psychology, T-junctions were described as a basis of monocular depth perception by the work of Kanizsa (Kanizsa, 1979). Later on, more computational works demonstrated the capability of T-junctions for depth estimation; to the best of our knowledge, one of the first attempts at depth ordering methods using T-junctions was performed by (Marr, 1982). Later on, an inspiring work of Nitzberg et al. (Nitzberg and Mumford, 1990; Nitzberg et al., 1993) proposed the so-called 2.1D sketch through a joint segmentation and depth estimation model. More recently, studies have been conducted using energy minimization approaches which use either explicit (Gao et al., 2007; Palou and Salembier, 2011) or implicit (Esedoglu and March, 2003) junction detection algorithms.

In addition to T-junctions, convexity is considered to be one of the most dominant cues for figureground organization (Kanizsa, 1979). A computational model for utilizing convexity has been developed for figure-ground organization in the recent past (Pao et al., 1999). Moreover, works on occlusion reasoning using Gestalt-based methods have used convexity as a complementary cue to T-junctions for a more robust relative depth estimation (Dimiccoli et al., 2008; Calderero and Caselles, 2013; Palou and Salembier, 2013). While it has been suggested that convexity affects human depth perception and is coded explicitly in the brain (Burge et al., 2010), the literature in computational models that use convexity is divided in this sense. In the works (Dimiccoli et al., 2008; Palou and Salembier, 2013), convexity is explicitly detected and coded, while in (Calderero and Caselles, 2013) this is done implicitly. The proposed approach shares with (Dimiccoli et al., 2008; 


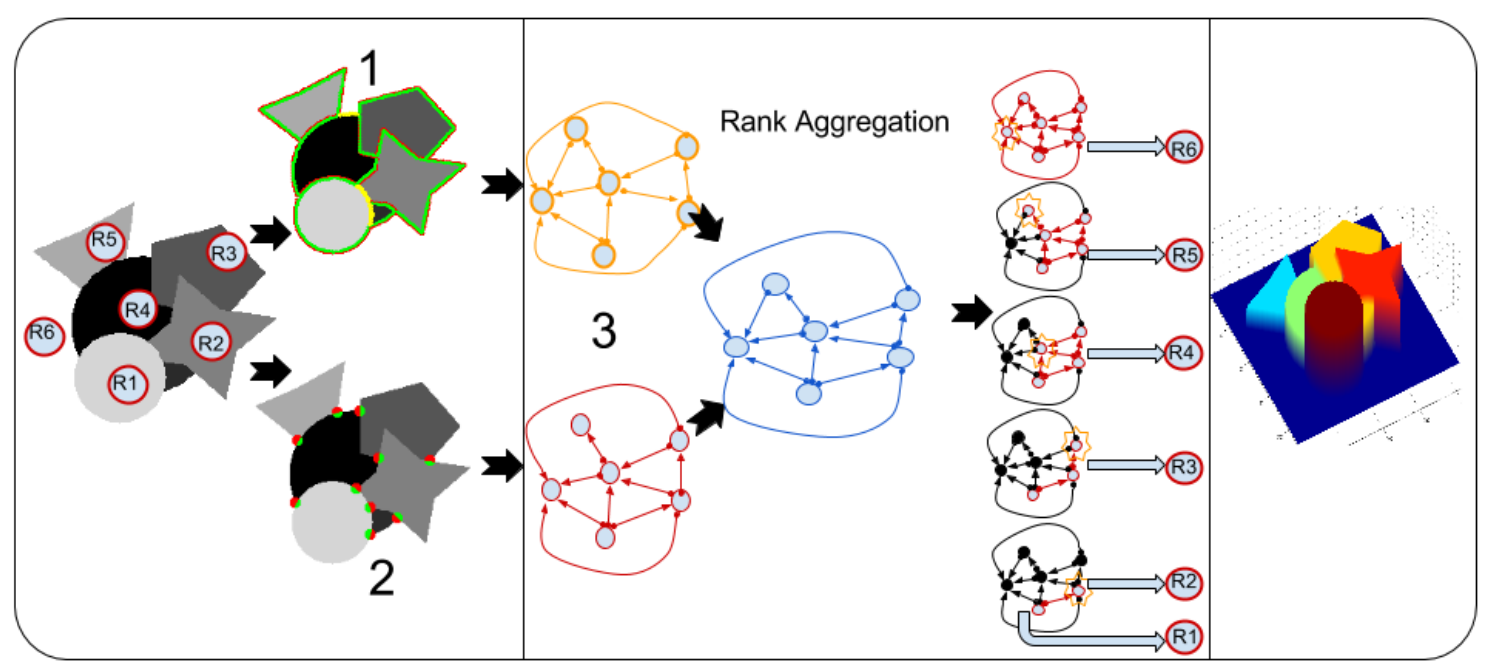

Figure 2: Diagram of the proposed method. From left to right: The segmented image; detected convexities cues (above) and T-junctions cues (below) and the local depth order from each cue inferred by the local cues (green areas are in front of red ones, whereas yellow indicates an inconclusive cue); global depth order extraction by rank aggregation on a graph whose nodes represent the different shapes and the directed edges indicate local depth orders; final result with global depth order illustrated as a depth map, where warmer color values indicate closer objects to the camera.

Palou and Salembier, 2013; Calderero and Caselles, 2013) the use of convexity and T-junctions cues. In order to integrate the partial depth orders suggested by the monocular depth cues we use a graph-based approach. Previous works (Dimiccoli and Salembier, 2009b; Palou and Salembier, 2013) also use a graph representation but need to reduce it to an acyclic graph and remove conflicts among different cues. In contrast, our work can directly handle conflicting transitive orders in the graph by using a rank-aggregationbased method (Basha et al., 2012), and obtain a globally consistent depth order. Here, transitive order is the order established by a path in the graph involving more than two nodes using the transitivity property mentioned in Section 1. A very recent work on depth layering using occlusion cues is the work of (Zeng et al., 2015) where convexity, T-junctions and a ground contact cue is used to obtain a depth order of the image. An energy minimization scheme is used to find the correct depth order which makes their method more complex and time consuming than our proposed method. Moreover, they have to make more restrictive assumptions to obtain the correct ground contact cue which limits their method to a smaller domain. As the method proposed by (Zeng et al., 2015) shows promising results and performs superior to other similar methods (Jia et al., 2012; Palou and Salembier, 2013), it has been used as a benchmark for evaluation of our proposed method. A comparative evaluation using the experimental setup in (Zeng et al., 2015) is presented in Section 4.

\section{PROPOSED METHOD}

We propose a method to extract a global depth order from a single image from an uncalibrated camera. The idea is motivated by studies showing human vision capability to integrate monocular depth cues to create a sensible depth perception. Given an input image, let us consider the set of its (segmented) shapes the notion of shape used in this paper will be clarified in Section 3.1.1. Then, a global depth order can be obtained following the steps below:

1. Determine a local depth order between each pair of adjacent shapes by analysing the convexity of their common boundaries.

2. Detect T-junctions and use a multi-scale feature to determine a local depth order between the shapes that meet at each T-junction.

3. Establish a global depth order by rank aggregation of the previous partial local orders.

Each step of the proposed method is detailed in the following sections. Figure 2 illustrates the different steps of the algorithm.

\subsection{Local Depth Cues Detection}

Local depth cues are extracted to establish a local depth order between neighbouring objects. In this work, convexities (L-junctions) and T-junctions are used for this purpose. We use a segmentation of 
the image as an input to the cue detection mechanism. In order to compute a local depth order in a manner that follows the human perception based on psychophysics studies (Kanizsa, 1979; McDermott, 2004; Burge et al., 2010), T-junctions and convexities must be treated in a different manner. Thus, an explicit detection of such depth cues is required. In the following, we explain how we detect both kind of junctions.

\subsubsection{Convexity Cue}

In this paper we propose a global convexity decision about each connected boundary between any two adjacent (segmented) regions in the image. The aim of this step is to determine which side of the boundary is the occluder and which side is the occluded, thus establishing a local depth order. Given the dead leaves model assumption, this cue can be used to infer the local depth order of the shapes that share a boundary.

To find the occluding region, we propose a method to determine which side of the boundary is closer to a convex shape. Figure 3 illustrates this process. Initially the segmented image is used to obtain the set of all the common boundaries between any two regions or objects in the image (Fig. 3, left image). For each connected common boundary, we consider its bounding box (shown in Fig. 3, middle-up). A connected common boundary divides the bounding box into two shapes (denoted by $S$ and $S^{\prime}$ in Fig. 3). The shape whose area is closer to the area of its convex hull (i.e. smaller red area in Fig. 3, middle-down) is considered more convex and assigned as the occluder ( $S$ in the example of Fig. 3). On the other hand, the complement shape ( $S^{\prime}$ in Fig. 3 ) is assigned as the occluded.

Let us notice that there is the possibility that a given boundary does not provide a conclusive depth cue. In other words, the convexity cue does not provide enough information to clarify which side is the occluder and which side is the occluded. This phenomenon appears, for instance, when the common boundary is either a straight line or a sinusoidal curve. To deal with such cases we define a criteria based on

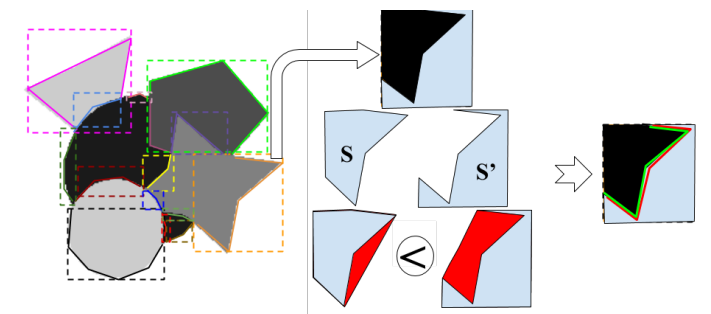

Figure 3: Illustration of the main steps of the convexity cue detector and the estimated local order where green areas are estimated to be in front of red areas. a threshold on our proposed global convexity measure of the connected boundary between two adjacent regions. This criteria is derived from the absolute difference between the convexity defect areas (red areas in Fig. 3) of the shapes ( $S$ and $S^{\prime}$ ). If this value is not significant enough (i.e. it is lower than a prescribed threshold $t h r_{C X}$ ) then these boundaries are considered inconclusive and will have no effect on the result. We define this threshold as $t h r_{C X}=L \cdot \pi \cdot t h r$, where $L$ is the length of the boundary and $t h r$ is a tuning parameter that controls the sensitivity of the criteria and is independent of the length of the boundary. Examples of such inconclusive boundaries for different values of $t h r$ can be found in Figure 4; namely, the figure displays examples for a smaller value of $t h r=0.0$ and a bigger value of $t h r=0.6$. In order to study the effect of this parameter, both on the local and global depth ordering, we present in Section 4 some experiments where the threshold $t h r$ is modified in the range of $[0.0,0.6]$ with step size of 0.05 .

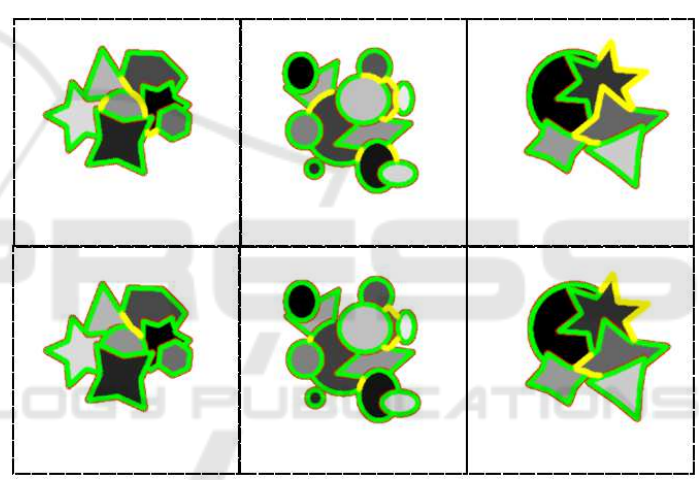

Figure 4: Illustration of modifying the value of $t h r_{C X}$ through the parameter $t h r$. Top row, $t h r=0.5$; bottom row, $t h r=0.15$. Decreasing $t h r$ leads to accepting more conclusive boundaries (less inconclusive boundaries in yellow).

\subsubsection{T-junctions Cue}

One of the pivotal depth cues used in this paper are T-junctions. T-junctions appear at the meeting points of three shapes boundaries and are related to occlusion configurations (see Figure 2). Two of the three regions present in the T-junction are separated by the stem of the T; these two regions are perceived to be partly occluded by the region which presents a larger section or angle. The latter region is then in front of the other two. Moreover, the angle of each object forming the junction must satisfy some criteria to be classified as a T-junction.

In this paper, we compute T-junctions using the method in (Caselles et al., 1999) where the authors gave a definition of T-junction which overcomes the difficulty of computing angles in a discrete image. 
They proposed an efficient algorithm which is mainly based on thresholding and computes junctions directly on the image without previous preprocessing or smoothing. The segmented image is used as an input to this method and the output is the locations of T-junctions.

The definition is based on the topographic map of an image $u: \Omega \in \mathbb{R}^{2} \rightarrow \mathbb{R}$ (in our case, the segmented image), that is, the family of the connected components of the so-called, level sets of $u,[u \geq \lambda]:=\{x \in$ $\Omega: u(x) \geq \lambda\}$, and on its boundaries, the so-called level lines. Here, $\lambda$ represents the gray level of the segmented image $u$. The set of level sets is invariant to monotonic non-decreasing illumination changes, a classical requirement in image processing and computer vision (Serra, 1986), and the level lines contain the boundaries of the parts of the physical objects projected on the image plane.In practice, the algorithm computes the T-junctions as all the pixels $p$ where two level lines meet and such that the area of the connected component of each of the bi-level sets $[u \leq \alpha]$, $[\alpha<u<\beta],[u \geq \beta]$, with $\alpha<\beta$, meeting at $p$ is big enough.

After detecting the location of T-junctions, for establishing a local depth ordering one could use some angle or area of the regions meeting at the T-junction, both of which have been used in the literature (Dimiccoli and Salembier, 2009a; Palou and Salembier, 2013). Problems arise when certain configurations of the cue lead to an inaccurate computation. One of the problems is related to the scale at which the depth cue is obtained.

Noise in the image can also lead to incorrect cues, so one could use larger scales but they are less discriminative in depth. To avoid these issues, we stem from the work by (Calderero and Caselles, 2013) to create a reliable multi-scale measure to establish a local depth order (according to human vision) at the located cues. To this end, features are formulated using the curvature of the level lines of the distance function of each connected component in the segmented image at different scales. The features are computed for each scale $s$ by adding the contribution from each connected component using the following formula:

$$
E_{s}(x)=\sum_{c=1}^{n c}\left(e^{\beta_{s}\left|K_{c, s}(x)\right|^{\gamma_{s}}}-1\right),
$$

where $K_{c, s}$ is the curvature of the level lines of the distance function to the connected component $c$ at scale $s, n c$ is the number of connected components at scale $s, \gamma_{s}$ and $\beta_{s}$ are scale-related parameters which are fixed as proposed in (Calderero and Caselles, 2013). In order to keep these features local and avoid overlapping with other boundaries, the distance function is clipped at a distance 5 . In order to generate a multiscale local feature we combine the local features according to (1) by computing an average of the normalized features at several scales, as in (Calderero and Caselles, 2013). In this work, we integrate the features from scales 1 to 5 . Figure 5 illustrates with an example the behaviour of this multi-scale features. As it can be seen in Fig. 5 right, the part of the cue that is perceptually closer to the observer has a higher multi-scale feature value.

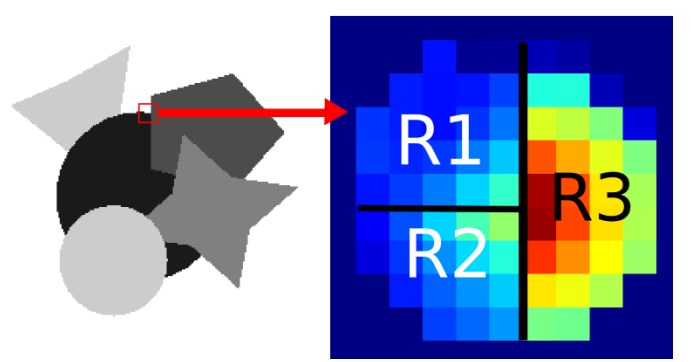

Figure 5: Multi-scale features obtained after averaging the features $E_{S}(1)$ of the first five scales.

Finally, to estimate the local depth order induced by a certain T-junction, first a representative value of the multi-scale depth features is computed for each region (e.g. R1, R2, and R3 in Fig. 5 right) in the neighborhood of the $\mathrm{T}$-junction given by a disk of radius 5. The representative value is computed by applying either the median or max operators on the features of the respective region (i.e. $\mathrm{R} 1, \mathrm{R} 2, \mathrm{R} 3$ ). In section 4 we compare the performance of both operators. The region with a higher representative local feature value is assigned to be in front of the other two neighboring regions ( $\mathrm{R} 3$ in front of $\mathrm{R} 1$ and $\mathrm{R} 2$ in the example of Fig. 5).

\subsection{From Local to Global Depth}

In order to establish a global depth order given by the local cues we use an approximation of rank aggregation (Dwork et al., 2001) similar to the one used in (Basha et al., 2012) for photo-sequencing. To do so, we construct a weighted graph $G(U, E)$ to represent the partial order between pairs of shapes (objects), which are represented in the graph by the nodes in $U$. The graph is constructed by placing a directional edge $e(i, j) \in E$ connecting the node $i$ to node $j$ if the local cues relating the objects suggest that object $i$ is in front of object $j$ (represented here by $i>>j$ ). The weight of the edge gathers up the local depth order cues. Each convexity cue indicates a depth order relation between two nodes (e.g. $i<<j$ ) and each Tjunctions indicates a relation between three nodes using two edges (e.g. $i<<j, i<<k$ ). The weight of 

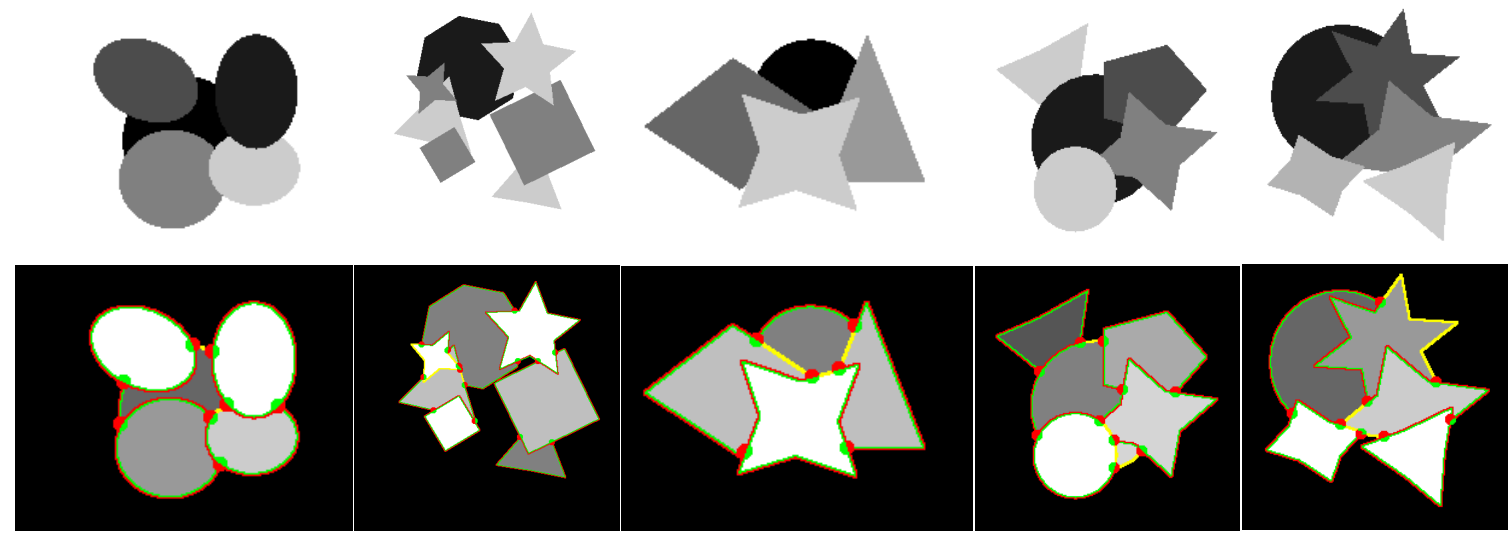

Figure 6: Experiments with synthetic images: estimated global depth ordering (brighter gray levels indicate closer objects). The automatically detected local depth cues, convexities and T-junctions establish a local depth order (green areas are estimated to be in front of red areas). Inconclusive convexity cues are marked in yellow.

the edge $e(i, j)$ between nodes $i$ and $j$ is proportional to the number of local cues indicating the local order $i>>j$, which can be interpreted as proportional to the number of votes for the local order $i>>j$. This weight corresponds to the probability that $i>>j$. In such a graph, a random walk after a sufficient time (in the steady state) will reach the sink of the graph (or of a sub-graph) which represents the object (or objects) perceptually furthest from the viewer. Repeating this process iteratively while in each iteration removing the sink node (or nodes) from the previous iteration will provide us with the global depth order. In particular the iteration number in which a set of nodes is removed reveals the global order of this set of nodes. For illustration of this process see Figure 2- step 3.

The steady state can be computed using an eigenvector analysis of $M$, the transition state matrix associated to the graph. The elements of $M$ are the probabilities of moving from one state (node) to another. To construct the matrix $M$ with non-negative entries, we initially form a matrix $V$ collecting the votes, where the rows and columns indices correspond to the index of each associated connected component. Thus, an image with $N$ shapes will produce an $N \times N$ matrix $V$. The $i, j$-th element of matrix $V, V(i, j)$, collects the number of votes (local cues) that agree with the partial ordering $i>>j$.

Once the matrix $V$ is filled, we compute the matrix $M$ which specifies the probability that $i>>j$. Firstly, the cycles of length two which may have been introduced by conflicting cues are removed. We follow the method proposed in (Basha et al., 2012) to resolve these conflicts. In particular, $M(i, j)=1-\frac{V(j, i)}{V(i, j)}$, and $M(j, i)=0$ if $V(i, j)>V(j, i)$. The rest of the cycles do not need to be removed since the rank aggregation method automatically solves them. Finally, the rows of $M$ are normalized to 1 in order to get transition probabilities.

\section{EXPERIMENTAL RESULTS}

This section presents three different experiments with different kind of data designed to evaluate and illustrate various aspects of the proposed method. An initial experiment is first presented as a proof of concept using synthetic images with the following parameters: $t h r=0.15$ for convexity cue detection, and median as T-junction feature operator. The goal of the second experiment is twofold: first, to present an experimental study of different parameter settings to find the best performance and fix the parameter values for the rest of the experiments and, second, to provide a quantitative comparison of the proposed method and the most recent state-of-the-art methods (Jia et al., 2012; Palou and Salembier, 2013; Zeng et al., 2015). This experiment is done using a dataset of 52 images proposed by (Zeng et al., 2015). For both the first and second experiments the ground truth segmentation is available, whereas in the third experiment the segmentation is done using an interactive tool (Santner et al., 2010).

Figure 6 illustrates the results of applying the proposed method to a small set of synthetic images. The first row shows the input images and the second row shows the global depth order images with convexity and T-junctions cues superimposed on them, respectively. The local depth order is illustrated in each cue, where green indicates the section perceived to be closer to the observer. As for global depth order, the grey values indicate global depth order, particularly the brighter areas are closer to the observer. As it can be seen all T-junction cues indicate a correct local depth order, whereas some of the convexity 


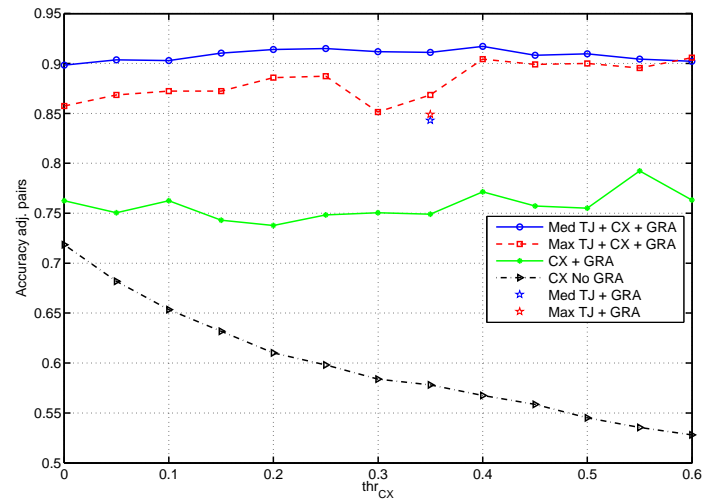

Figure 7: Accuracy of local depth order between adjacent pairs of shapes.

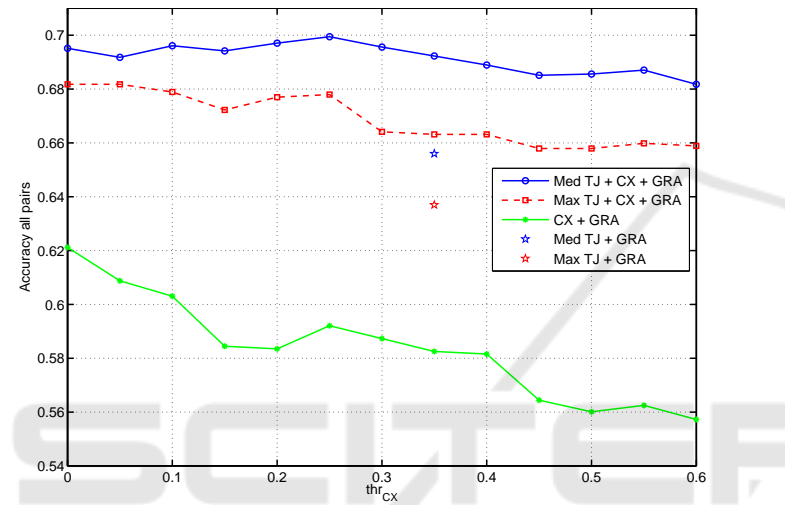

Figure 8: Accuracy of global depth order between all pairs of shapes.

Table 1: Depth Order accuracy.

\begin{tabular}{|l||l|l|}
\hline & $\begin{array}{l}\text { adj. } \\
\text { pairs }\end{array}$ & $\begin{array}{l}\text { all } \\
\text { pairs }\end{array}$ \\
\hline (Jia et al., 2012) & 79.84 & 29.88 \\
(Palou and Salembier, 2013) & 43.85 & 43.56 \\
(Zeng et al., 2015) & 82.66 & $\mathbf{8 4 . 6 0}$ \\
\hline Our method: CX+GRA & 74.83 & 59.21 \\
Our method: TJ+GRA & 84.39 & 65.3 \\
Our method: TJ+CX+GRA & $\mathbf{9 1 . 4 9}$ & 69.94 \\
\hline
\end{tabular}

cues are incorrect or inconclusive (marked as yellow). However, the T-junctions cues are able to compensate these errors and create a globally consistent depth order that complies with human depth perception.

In the first part of the second experiment the proposed method is evaluated under different parameter settings with the dataset proposed by (Zeng et al., 2015). Figures 7 and 8 illustrate these results. The horizontal axis denotes the parameter $t h r$ that defines the threshold $t h r_{C X}=L \cdot \pi \cdot t h r$ applied to the difference of defect areas. Then, as the values in the horizontal axis increase the threshold $t h r_{C X}$ increases and more boundaries become inconclusive, meaning that the sensitivity for detecting global convex boundaries decreases (see also Fig. 4). In Figure 7 the vertical axis indicates the accuracy as the percentage of pairs of adjacent shapes which have been assigned a correct local depth order. Whereas in Figure 8 the vertical axis indicates the accuracy as the percentage of pairs of all shapes which have been assigned a correct global depth order. These accuracy measures are identical to the measures of performance evaluation in (Zeng et al., 2015). The legend of Figures 7 and 8 indicate the operator for the T-junction (median or max), the type of local cue (T-junction (TJ), convexity (CX) or both), and whether or not a global rank aggregation was used ("GRA" or "No GRA"). Both Figures 7 and 8 indicate that the best performance is achieved when the depth order induced by the Tjunction cues is computed using the median operator and is combined with the depth order induced by the convexity cue using rank aggregation, denoted as "Med TJ $+C X+G R A$ ". Thus, achieving a top performance of $91.49 \%$ accuracy in local depth order estimation and $69.94 \%$ in global depth order estimation. On the other hand, using the max operator slightly decreases the performance to $89 \%$ and $67.8 \%$ for local and global depth estimation, respectively.The decrease in accuracy of the global order with respect to the local order can be explained by the fact that the proposed method can only infer depth relations between objects connected by a path in the graph. It should also be noted that the performance of the max operator is slightly less stable. Further, it can be seen that the contribution of T-junctions is significant for both global and local depth estimation as they improve the performance compared to when only convexities are used (16\% increase for local depth estimation and $19 \%$ increase in accuracy of global depth estimation). The blue and red stars in the Figures 7 and 8 highlight the performance of using only T-junctions (the parameter $t h r$ does not affect this computation). As expected, T-junctions seem to be a more reliable cue than convexities as they consistently achieve a higher accuracy. Figure 7 illustrates how the global integration of convexity cues using rank aggregation improves the performance of local depth estimation between adjacent pairs of shapes, namely, the performance increases from $59 \%$ to $75 \%$. Finally, observing the two lower curves the in Figure 7 we can see that, while the accuracy of " $C X+N o G R A$ " decreases as the threshold increases, the accuracy of " $C X+G R A$ " remains relatively stable. This indicates that most of the convexity cues in the dataset are conclusive (i.e. comply with human depth perception) and increasing the threshold will lead to less cues and thus less accuracy. However, it is interesting to note 

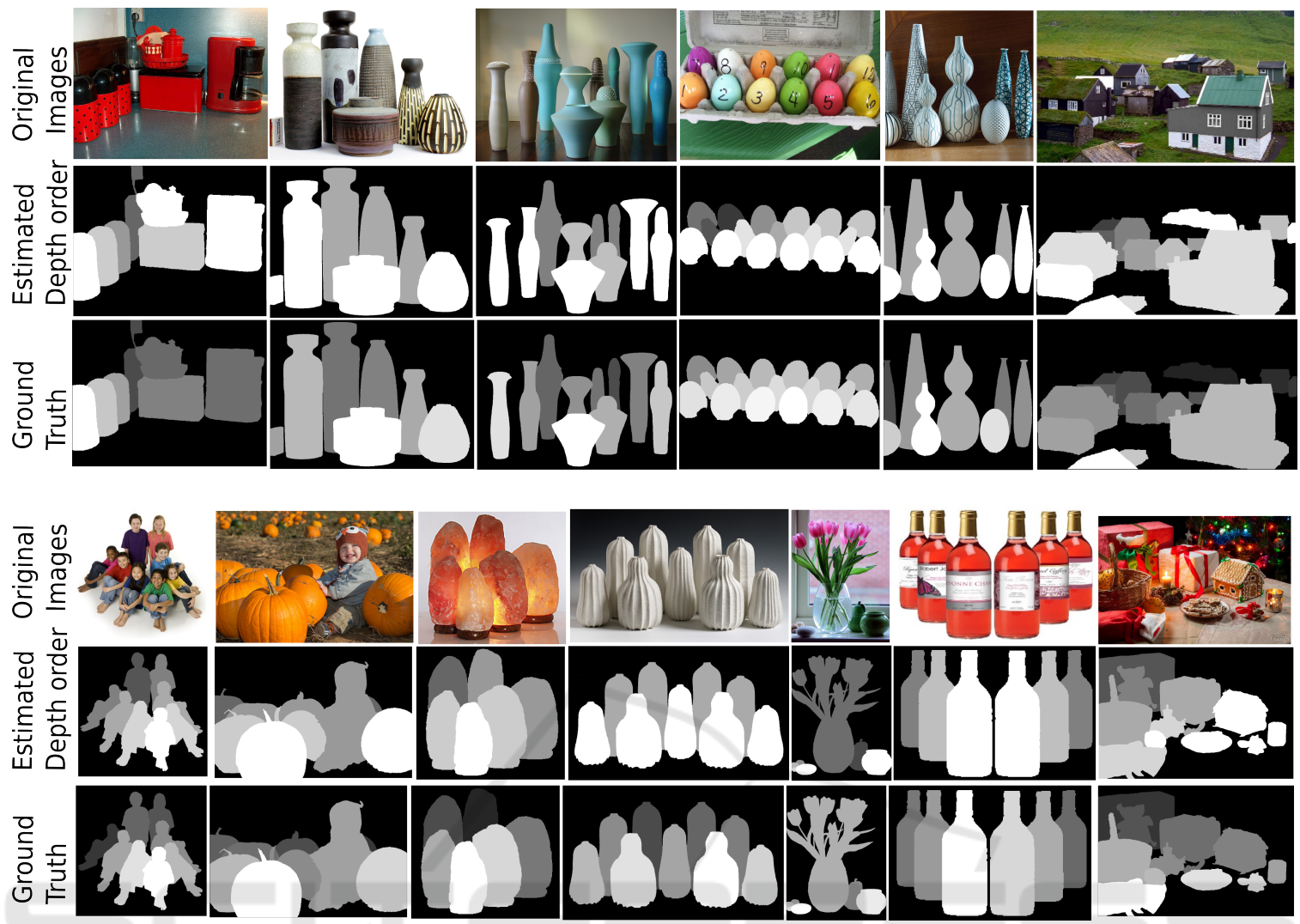

Figure 9: Depth ordering results using the proposed method on near-view scenes from the dataset by (Zeng et al., 2015).

that the global integration is able to compensate for the removal of cues that did not satisfy the threshold and stabilize the performance.It can be seen that the best operation point for the threshold of the global convexity is the mid-range value $t h r=0.25$, where the average of the two accuracy measures is the highest. While the effect of $t h r$ is not significant it leads to a slight increase in the performance of the global depth estimation (see Figure 8).

In the second part of the second experiment, the proposed method is compared with the state-of-theart (Jia et al., 2012; Palou and Salembier, 2013; Zeng et al., 2015) with the accuracy measures presented in (Zeng et al., 2015). According to the results obtained in the previous analysis, we fix the parameters to the following values: $t h r=0.25$ and median as the operator in the depth order estimated from the T-junctions. To this end, we follow the experimental setup suggested by (Zeng et al., 2015) on their proposed depth ordering dataset. The results in Table 1 show that using a combination of T-junction and convexity cues achieves the highest performance. As it can be seen, the proposed method outperforms all of the state-ofthe-art methods in the adjacent pairs case and, in the all pairs case, the proposed method performs superior to (Jia et al., 2012) and (Palou and Salembier, 2013) but falls short of (Zeng et al., 2015). This is mainly,as previously noticed, due to the fact that our proposed method cannot infer depth relations between objects that are not are not connected with a path in our graph i.e. there are no transitive relations to be used to infer a global depth order. In contrast, the method by (Zeng et al., 2015) uses the ground contact as an extra cue to order shapes when other cues (T-junction, convexity) are not present. This could be added in order to make the constructed graph more connected. In other words there are more transitive relations (paths) that can be used to infer depth relations. All the previous experiments were carried out using Matlab on an eight core $3.5 \mathrm{GHz}$ Core i7 processor with an average computation time of 8.4 seconds per image.

Finally, to show how the proposed method may be used as a real world application, the interactive segmentation tool (Santner et al., 2010) has been used to segment some images from the Berkeley dataset (Martin et al., 2001) and the global depth order of the segmented objects is estimated with the proposed method. As it can be seen in Figure 10 the order of the segmented objects is correct in most of the cases. 


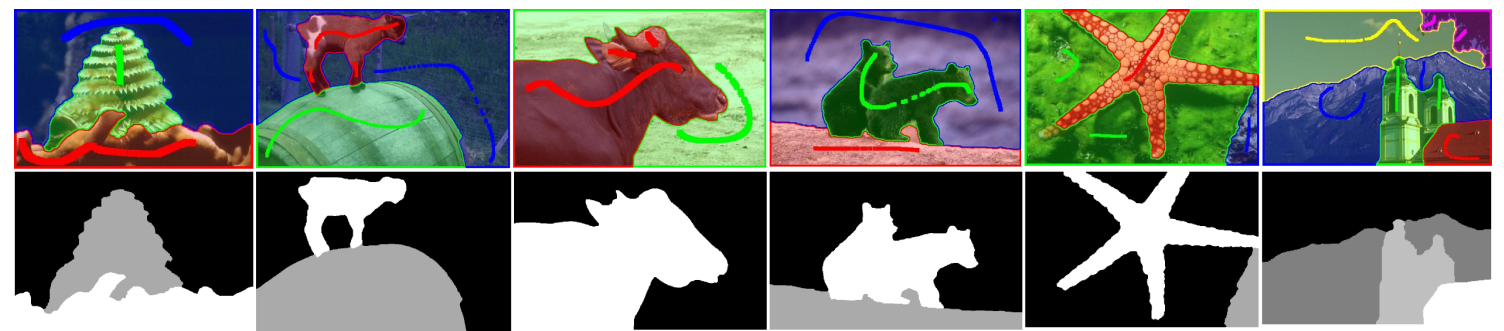

Figure 10: Using interactive segmentation (Santner et al., 2010) and the proposed method to create a depth ordering of objects in the scene.

\section{LIMITATIONS AND ASSUMPTIONS}

Estimating depth from a single image is a very challenging and under-determined problem. It is necessary to make suitable assumptions to make the problem tractable. Our first assumption is that a good segmentation is available where the boundaries of the segmentation regions coincide with the actual object boundaries. As the method is based on a convexity cue defined on boundaries and T-junctions (which are points at the intersection of boundaries), a deficient segmentation leads to significant depth artifacts in the estimated depth order. A second limitation may be noticed in one of the examples in Figure 11: the one in box 1 of the left image. The T-junction and convexity cues that are detected on the ground contact of the object indicate incorrect depth order. In some cases, there exist other cues that compensate for these mistakes, either directly or indirectly using the transitivity property of the graph. However, this is not the case in the aforementioned example. Another limitation is inaccuracies in our convexity detector which can be seen in Figure 11 box 2, a misinterpretation of convexities in cases where a long narrow shape is next to two concavities. Figure 11 box 3 shows the bias of the proposed method to interpret small convex objects to be in front of their neighbouring shapes (this may happen also in visual holes, such as windows or arch bridges). A more general limitation is that objects in the scene should be approximated with fronto-parallel planes to the camera. When this assumption does not hold it may lead to misinterpretation of local cues and thus misestimation in the order of objects. An example of this can be found in Fig. 11 right, box 4. In this case, since the two objects sharing the same border cannot be approximated with fronto-parallel planes, the algorithm misestimates the depth order. Fortunately, in some cases there are solutions to deal with the aforementioned limitations. The non-fronto parallel problem can be resolved by ground separation in simple cases. In cases where there are more than one non-fronto parallel planes in the image, a geometric context method, based for instance on surface normal extraction, may be used to guide the depth estimation. The problem related to visual holes can be addressed using a semantic labelling method that is able to identify the visual hole; for example by classifying areas like the sky which are always in the back.

\section{DISCUSSION AND CONCLUSION}

Inspired by the human vision capability to perceive depth using monocular cues, we proposed a method for the detection and integration of $\mathrm{T}$-junction and convexity cues that is able to obtain a globally consistent depth order. The proposed method computes partial depth orders using multi-scale features, then, integrates them using a rank aggregation method that resolves conflict. This allows to simultaneously compensate for incorrect partial depth orders introduced by invalid cues and also harnesses the transitivity between the cues to obtain a global order from partial orders. The proposed method is applicable to any scene that complies with the dead leaves model and does not require training. For future work we propose to extend the method to images containing non frontoparallel objects using other monocular and binocular cues that may be integrated in the rank aggregation step as additional votes for partial depth orders.

\section{ACKNOWLEDGEMENTS}

The authors acknowledge partial support by MICINN project, reference MTM2012-30772, and by GRC reference 2014 SGR 1301, Generalitat de Catalunya. 


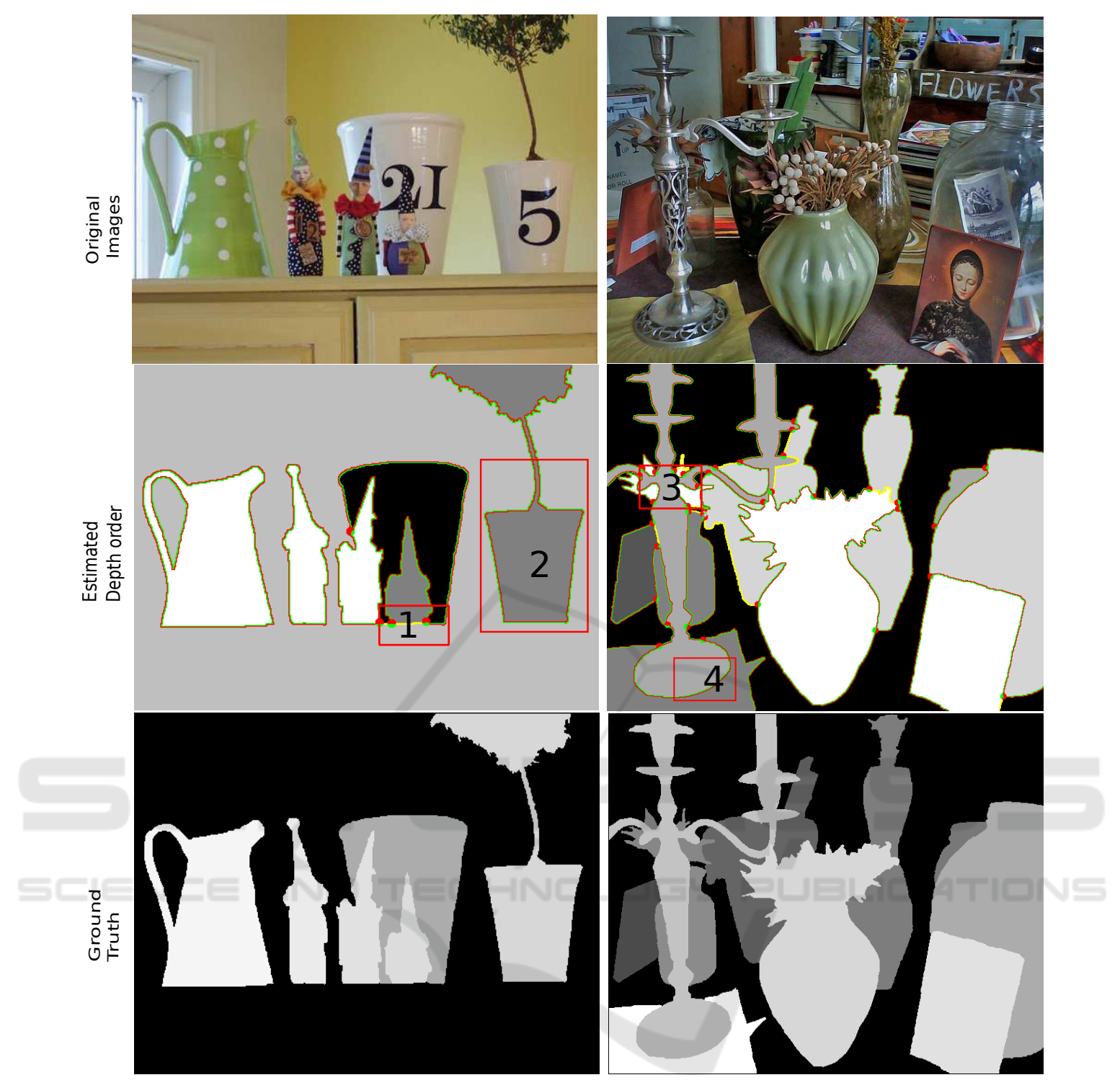

Figure 11: Due to some limitations of the proposed approach, the violation of certain assumptions leads to errors in the estimated depth order which have been delimited with red boxes (see Section 5 for a detailed explanation of these problems).

\section{REFERENCES}

Basha, T., Moses, Y., and Avidan, S. (2012). Photo sequencing. In Computer Vision-ECCV 2012, pages 654-667. Springer.

Burge, J., Fowlkes, C., and Banks, M. (2010). Naturalscene statistics predict how the figure-ground cue of convexity affects human depth perception. The Journal of Neuroscience, 30(21):7269-7280.

Calderero, F. and Caselles, V. (2013). Recovering Relative Depth from Low-Level Features Without Explicit Tjunction Detection and Interpretation. International Journal of Computer Vision, 104:38-68.

Caselles, V., Coll, B., and Morel, J. (1999). Topographic maps and local contrast changes in natural images. International Journal of Computer Vision, 33(1):5-27.

Chen, X., Li, Q., Zhao, D., and Zhao, Q. (2013). Occlusion cues for image scene layering. Computer Vision and Image Understanding, 117(1):42-55.

Dimiccoli, M., Morel, J.-M., and Salembier, P. (2008). Monocular depth by nonlinear diffusion. In Computer Vision, Graphics \& Image Processing, 2008. ICVGIP'08. Sixth Indian Conference on, pages 95102. IEEE.

Dimiccoli, M. and Salembier, P. (2009a). Exploiting t-junctions for depth segregation in single images. Acoustics, Speech and Signal ..., pages 1229-1232.

Dimiccoli, M. and Salembier, P. (2009b). Hierarchical 
region-based representation for segmentation and filtering with depth in single images. Image Processing (ICIP), 2009 16th , 1:3533-3536.

Dwork, C., Kumar, R., Naor, M., and Sivakumar, D. (2001). Rank aggregation methods for the web. In Proceedings of the 10th international conference on World Wide Web, pages 613-622. ACM.

Eigen, D., Puhrsch, C., and Fergus, R. (2014). Depth map prediction from a single image using a multi-scale deep network. In Advances in Neural Information Processing Systems, pages 2366-2374.

Esedoglu, S. and March, R. (2003). Segmentation with depth but without detecting junctions. Journal of Mathematical Imaging and Vision, 18(1):7-15.

Gao, R., Wu, T., Zhu, S., and Sang, N. (2007). Bayesian inference for layer representation with mixed markov random field. Energy Minimization Methods in Computer Vision and Pattern Recognition, 4679:213-224.

Guzmán, A. (1968). Decomposition of a visual scene into three-dimensional bodies. In Proceedings of the December 9-11, 1968, fall joint computer conference, part I, pages 291-304. ACM.

Hoiem, D., Efros, A., and Hebert, M. (2011). Recovering Occlusion Boundaries from an Image. International Journal of Computer Vision, 91(3):328-346.

Jia, Z., Gallagher, A., Chang, Y., and Chen, T. (2012). A learning-based framework for depth ordering. In Computer Vision and Pattern Recognition (CVPR), 2012 IEEE Conference on, pages 294-301. IEEE.

Kanizsa, G. (1979). Organization in vision: essays on Gestalt perception. NY, Praeger.

Liu, B., Gould, S., and Koller, D. (2010). Single image depth estimation from predicted semantic labels. In Computer Vision and Pattern Recognition (CVPR), 2010 IEEE Conference on, pages 1253-1260. IEEE.

Malik, J. (1987). Interpreting line drawings of curved objects. International Journal of Computer Vision, 73403.

Marr, D. (1982). Vision: A computational approach. San Francisco: Free-man \& Co.

Martin, D., Fowlkes, C., Tal, D., and Malik, J. (2001). A database of human segmented natural images and its application to evaluating segmentation algorithms and measuring ecological statistics. In Proc. 8th Int'l Conf. Computer Vision, volume 2, pages 416-423.

Matheron, G. (1968). Modèle séquentiel de partition aléatoire. Technical report, CMM.

McDermott, J. (2004). Psychophysics with junctions in real images. Perception, 33(9):1101-1127.

Nitzberg, M. and Mumford, D. (1990). The 2.1-d sketch. In Computer Vision, 1990. Proceedings, Third International Conference on, pages 138-144. IEEE.

Nitzberg, M., Mumford, D., and Shiota, T. (1993). Filtering, segmentation, and depth, volume 662. Lecture notes in computer science, Springer.

Palou, G. and Salembier, P. (2011). Occlusion-based depth ordering on monocular images with binary partition tree. In Acoustics, Speech and Signal Processing (ICASSP), 2011 IEEE International Conference on, pages 1093-1096. IEEE.
Palou, G. and Salembier, P. (2013). Monocular depth ordering using t-junctions and convexity occlusion cues. IEEE Transactions on Image Processing, 22(5):19261939.

Pao, H., Geiger, D., and Rubin, N. (1999). Measuring convexity for figure/ground separation. In Computer Vision, 1999. The Proceedings of the Seventh IEEE International Conference on, volume 2, pages 948-955. IEEE.

Rubin, N. (2001). Figure and ground in the brain. Nature Neuroscience, 4:857-858.

Santner, J., Pock, T., and Bischof, H. (2010). Interactive multi-label segmentation. In Proceedings 10th Asian Conference on Computer Vision (ACCV), Queenstown, New Zealand.

Saxena, A., Chung, S. H., and Ng, A. Y. (2008). 3-d depth reconstruction from a single still image. International journal of computer vision, 76(1):53-69.

Serra, J. (1986). Introduction to mathematical morphology, volume 35(3). Elsevier.

Zeng, Q., Chen, W., Wang, H., Tu, C., Cohen-Or, D., Lischinski, D., and Chen, B. (2015). Hallucinating stereoscopy from a single image. In Computer Graphics Forum, volume 34, pages 1-12. Wiley Online Library.

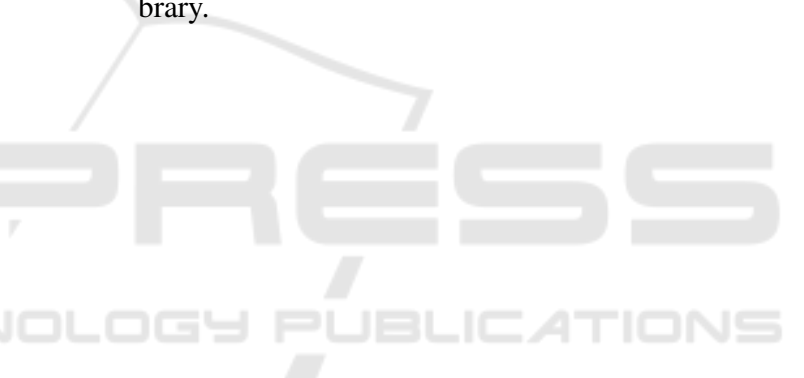

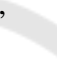

